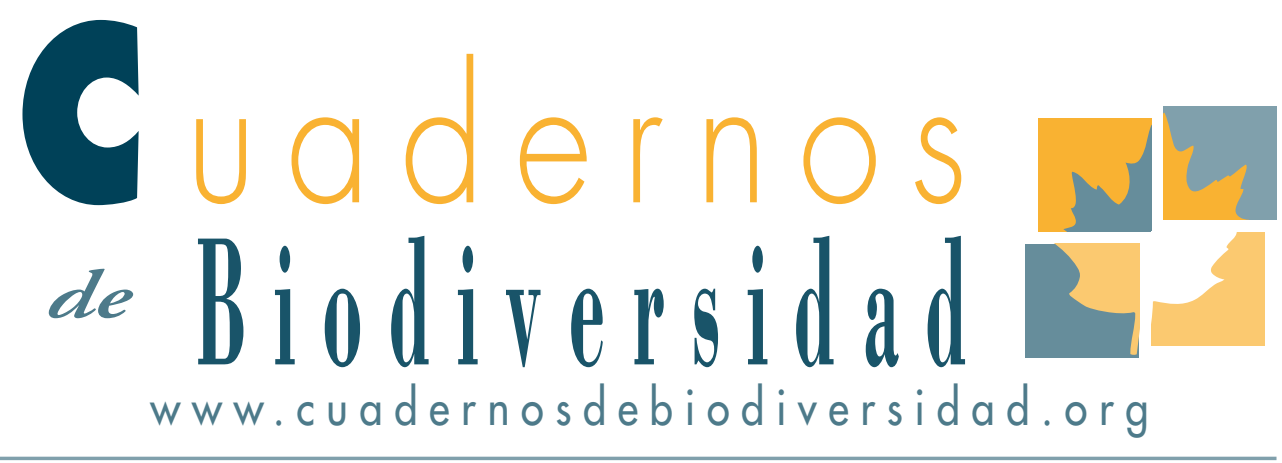

\title{
Ecología química y perspectivas de su aplicación en la conservación de la biodiversidad
}

\section{Vieyle Cortez}

Red de Ecoetología, Instituto de Ecología, A.C., Carretera a Coatepec No. 35 i, El Haya, A. P. 63, Xalapa, 9io70, Veracruz, México. E-Mail: Vieyle@Gmail.Com

\section{ABSTRACT}

Chemical ecology comprises the study of interactions of organisms with their environment that are mediated by the chemicals they produced. In general, chemicals that mediate interactions between organisms are called semiochemicals. Semiochemicals are divided into two major groups depending on whether the interactions are intraspecific (pheromones) or interspecific (allelochemics). The development of chemical ecology has been stimulated by the interest in knowing the details of the chemical interactions between organisms and the benefit of exploring new semiochemicals. In recent years, studies of chemical ecology have contributed to the conservation of biodiversity, demonstrating how valuable this can be in many ways. In the present context, chemical ecology studies open up a field of knowledge for the conservation of the olive ridley sea turtle (Lepidochelys olivacea), specie in danger of extinction. This study provides a powerful basis for the development of a new control method for reducing the impact of the beetle Omorgus soberosus Fabricius (Coleoptera: Trogidae), which significantly affects the survival of eggs of L. olivacea on Santuario La Escobilla Beach in Oaxaca, Mexico.

\section{KEY WORDS:}

Chemical ecology, semiochemicals, conservation, biodiversity. 


\section{INTRODUCCIÓN}

La Ecología Química es una de las disciplinas más recientes cuyo enfoque dentro de la investigación científica, está teniendo un gran impacto en las áreas de ciencias químicas y biológicas. Un trabajo de referencia sobre éste nuevo enfoque científico fue el de Erlich y Raven (1965), donde se aborda la química de los metabolitos secundarios de las plantas y la interacción ecológica entre especies de mariposas mediada por estos productos naturales. Así, en el siglo XX surge la ecología química, conduciendo al estudio de las sustancias químicas involucradas en las interacciones ecológicas intra e interespecíficas de organismos vivos (Ruther et al., 2002). Se acuña el término de "semioquímicos", del griego semion que significa marca o señal, para nombrar las sustancias químicas involucradas en las distintas interacciones ecológicas (Law y Regnier, 1971). Recientemente, Dicke y Sabelis (1988) propusieron el término "infoquímicos", teniendo en cuenta el carácter perjudicial o beneficioso, desde el punto de vista evolutivo, de las sustancias químicas en las interacciones entre seres vivos. Infoquímicos son compuestos que, dentro de un contexto natural, transmiten información entre individuos produciendo en el receptor una respuesta de comportamiento o fisiológica. Ésta puede ser ventajosa o no, desde un punto de vista adaptativo, a cualquiera de los integrantes o a ambos. El término infoquímico involucra el criterio de emisor, el cual no estaba considerado en la definición de semioquímico, en la que el elemento fundamental es el organismo productor. Sin embargo, la discusión del término continúa, por lo que Hick y colaboradores (1999) publicaron una revisión donde se justifica que el término más apropiado es el de semioquímicos.

\section{SEMIOQUÍMICOS}

Los semioquímicos son compuestos que se sintetizan a partir de rutas degradativas del metabolismo primario y sin tener un rol específico en éste. En la biosíntesis intervienen cientos de miles de enzimas especializadas y en la mayoría de los casos es un sustrato y una enzima para un compuesto. Sin embargo, puede ocurrir la síntesis de muchos productos por una sola enzima a partir de varios precursores o menos frecuentemente de un solo sustrato. Asimismo, una enzima puede catalizar una función química similar en el mismo sustrato, o por el contrario, catalizar una misma función química pero en diferente sustrato (Anaya et al., 2001). Esta red de síntesis da origen a muchos compuestos nuevos y rutas metabólicas nuevas, que dan lugar a una diversidad química que desempeña un rol importante en la historia evolutiva de las especies.

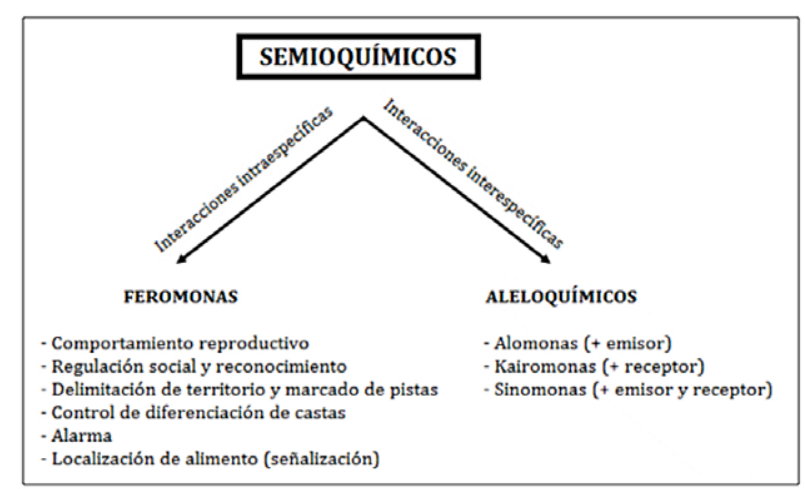

Figura 1. Clasificación de los semioquímicos

En los semioquímicos se pueden reconocer dos grandes grupos (Fig. 1): 1) las feromonas (del griego phereum, llevar; horman, excitar o estimular), que median interacciones entre organismos de la misma especie confiriendo ventajas de adaptación (o adaptativas) al organismo emisor (feromona $+/$-), al organismo receptor (feromona $-/+$ ) o a ambos (feromona $+/+$ ). De hecho, la diversidad de respuestas fisiológicas y de comportamiento inducida por la recepción de mensajes químicos en un organismo y emitida por otros organismos de la misma especie, pudo ser un factor principal en la evolución de los niveles de sociabilidad en insectos. 2) los aleloquímicos, que actúan a nivel interespecífico y que se clasifican según el costo-beneficio para cada uno de los organismos interactuantes. De este modo, Alomonas dan ventajas desde el punto de vista adaptativo al organismo que la produce y desventajas al organismo que la recibe. Kairomonas son favorables, desde el punto de vista adaptativo, para el organismo receptor. Sinomonas inducen una respuesta 
conductual o fisiológica que es favorable, desde el punto de vista adaptativo, para ambos organismos (Norlund y Lewis, 1976; Blum, 1996). Una parte elemental de estas interacciones está relacionada con la comunicación química, el modo primario de transferir información en muchos grupos de organismos, y es importante mencionar que dependiendo del contexto, un mismo compuesto químico puede pertenecer a categorías distintas de semioquímicos. La eficiencia de estas sustancias semioquímicas en la comunicación química dependerá de varias propiedades físico-químicas, entre ellas, la naturaleza química, la solubilidad, la volatilidad, el tiempo de vida en el ambiente, entre otras (Blum, 1981). Así, los estudios en ecología química han permitido reconocer que la comunicación química es uno de los atributos fundamentales para comprender el significado biológico de la vida.

Una extensa variedad de semioquímicos han sido identificados como productos exocrinos de artrópodos y se ha destacado la versatilidad biosintética que poseen estos invertebrados para producirlas (Blum, 1981). En muchos organismos la defensa química está constituida por muy pocos compuestos activos, pudiendo también existir una gran heterogeneidad de semioquímicos que equivalen a una gran defensa química y todos, o la mayoría de éstos, son o han sido adaptativos (Dettner, 1987). En ambos casos, se debe enfatizar la importancia de la variación cuantitativa y cualitativa, la especificidad de los semioquímicos, solos o en mezclas. Asimismo, es necesario entender tanto la evolución de los compuestos químicos, como la evolución de su interacción con los organismos. Las funciones de los semioquímicos y su amplia diversidad son el resultado de complejas y dinámicas interacciones biológicas. En la actualidad se han esclarecido múltiples y complejas interacción químicas entre organismos, lo que ha permitido entender mejor a la naturaleza y los procesos biológicos. Por lo tanto, la determinación de los diferentes roles de estos compuestos constituye uno de los retos reales en el estudio de la ecología química.

Para proporcionar un sentido de la inmensa diversidad química que existe en los organismos, uno puede consultar la "Pherobase" (http://www. pherobase.net), un catálogo virtual que contiene información de semioquímicos en insectos. Esta base de datos consiste en un listado de especies, designaciones taxonómicas, referencias de la literatura y lo más importante las estructuras químicas ligadas a las especies que las producen y su función. La diversidad estructural y la fuerte bioactividad de muchos de estos semioquímicos los convierten en excelentes candidatos para múltiples usos, por ejemplo en la industria farmacéutica (Caporale, 1995) o en el control de plagas (Heuskin et al., 2011). En algunos compuestos ya se han comprobado sus usos de interés humano, pero muchos más aguardan a su descubrimiento.

\section{UN CASO DE ESTUDIO DE ECOLOGIAA QUIMICA EN LA CONSERVACIÓN DE LA TORTUGA GOLFINA LEPIDOCHELYS OLIVACEA}

Recientemente, se ha demostrado la importancia de entender la visión multidisciplinaria de la ecología química en la provisión de un marco conceptual dentro de los procesos que amenazan la conservación de especies y ecosistemas. Dentro de este contexto y dentro de un marco de investigación emprendido por la Comisión Nacional de Áreas Naturales Protegidas (México) en colaboración con el Instituto de Ecología, A.C. (México), el CIBIO de la Universidad de Alicante (España) y la Universidad Politécnica de Linares (España), se llevó a cabo un estudio en ecología química para la conservación de la tortuga golfina Lepidochelys olivacea, especie vulnerable (UICN).

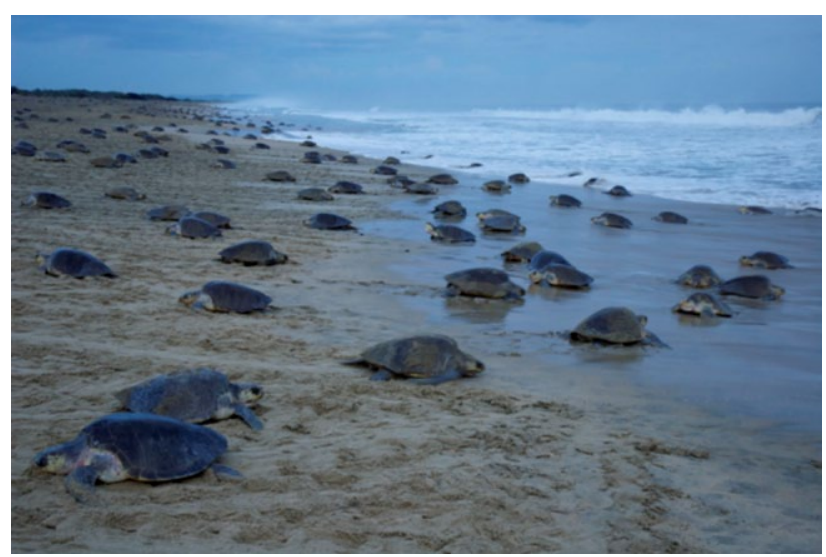

Figura 2. Área de anidación de la tortuga golfina Lepidochelys olivacea en el Santuario Playa La Escobilla, Oaxaca, México 
Desde 1987 se ha reportado una gran mortalidad de embriones de la tortuga $L$. olivacea en el Santuario Playa La Escobilla, Oaxaca, México (Fig. 2), el área de anidación más importante de esta tortuga a nivel mundial (López y Aragón 1994). El escarabajo Omorgus suberosus Fabricius (Coleoptera: Trogidae) es uno de los principales factores de mortandad de huevos de la tortuga marina. La infestación masiva por el escarabajo, al parecer está relacionada con la densidad y la distribución espacial de los nidos de la tortuga en el Santuario (López-Reyes y Harfush; 2000, Halffter et al., 2009).

Omorgus suberosus, a diferencia de las pocas especies de Trox y Omorgus con nidificación conocida, pierde los comportamientos larvarios agonísticos y territoriales al atacar recursos alimentarios concentrados. Esto lo convierte en una plaga potencial, ya que esta especie (a diferencia de otros Trogidae) puede responder con un rápido aumento de población (sin las barreras comportamentales antes señaladas), en cualquier circunstancia en la que se presente un alimento proteico abundante y concentrado.

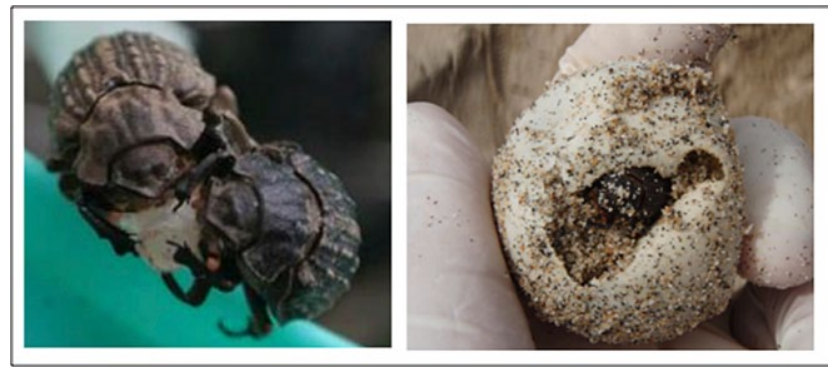

Figura 3. Omorgus suberosus: (izquierda) pareja de escarabajos, derecha) adulto alimentándose dentro de un huevo de Lepidochelys olivacea

Ejemplos son el ataque a los nidos de iguana (Onelopus scelbstriatus), el ataque masivo a puestas (ootecas) de langosta migratoria (Schistocerca paranensis) y el ataque a nidos de tortuga (Chelonia mydas) en las islas Galápagos. Consideramos que estos casos constituyen claramente procesos adaptativos muy recientes y localizados, que son respuesta a ofertas alimenticias concentradas y excepcionales.

Los resultados obtenidos hasta la fecha sugieren que $O$. suberosus presenta características de plaga al actuar como depredador tanto de huevos "vivos" como a "muertos" en condiciones naturales y de laboratorio. Por ello se ha recurrido a diversos méto- dos no específicos de captura directa para su control, aunque hasta el momento no se ha encontrado un método selectivo y efectivo. La razón principal es que no se cuenta con el conocimiento básico de su comportamiento y comunicación química. Dichos conocimientos resultan indispensables para el planteamiento de métodos y estrategias en el manejo de poblaciones de $O$. suberosus como insecto plaga. Frente a este escenario surgió la necesidad de contar con mayor información acerca de los aspectos básicos de comportamiento de $O$. suberosus y encontrar una alternativa específica para su control.

En general, no existen investigaciones acerca de compuestos volátiles en especies de los coleópteros Trogidae. Por ello, se realizó un análisis de los semioquímicos que intervienen en el comportamiento de localización de los nidos de la tortuga L. olivacea y en la agregación de $O$. suberosus, tales como feromonas y/o kairomonas. En este proyecto se plantearon cuatro etapas con el objetivo de sustentar el desarrollo de un sistema de monitoreo y trampeo para el control de $O$. suberosus en los nidos de la tortuga marina L. olivacea, en playa La Escobilla, Oaxaca. En su primera etapa, se llevó a cabo la extracción de los volátiles emitidos por la fuente de alimento (cadáveres de tortugas, huevos en descomposición, hevos frescos y huevos recién puestos) y muestras de las feromonas emitidas por los escarabajos de $O$. suberosus. Para ello, se utilizó un método novedoso y efectivo de extracción de volátiles conocida como microextracción con barra adsorbente "Twister" (SBSE). En su segunda etapa, se realizó el análisis de los compuestos volátiles de las muestras mediante un sistema de Desorción Térmica acoplado a Cromatografía de Gases-Espectrometría de Masas (TD-GC $\neg-\mathrm{MS}$ ). Los semioquímicos identificados hasta la fecha presentan antecedentes de actividad biológica en diversos insectos. Como una tercera etapa, se realizaron ensayos electrofisiológicos (EAG) y de olfatometría, como una herramientas para la identificación de los semioquímicos activos para O. suberosus. Los ensayos de EAG permitieron obtener un indicio de la actividad biológica de los compuestos identificados en los extractos volátiles mediante el registro las señales eléctricas generadas por las antenas de ambos sexos de $O$. suberosus ante los diversos estímulos. La respuesta conductual 
de hembras y machos de O. suberosus se evaluó mediante ensayos de olfatometría probando los compuestos identificados con actividad biológica. Los bioensayos mostraron que los individuos de $O$. suberosus son atraídos por volátiles liberados por la fuente de alimento y de sus congéneres. Dichos compuestos podrían tener una efectiva actividad en condiciones de campo como un método para solucionar el problema que ocasiona $O$. suberosus en la depredación de huevos de la tortuga $L$. olivacea. Como una última etapa se está desarrollando un sistema Trampa + Atrayente, en el Santuario Playa La Escobilla, Oaxaca, con el propósito de evaluar en forma preliminar el desempeño de los distintos compuestos estudiados según la actividad biológica demostrada en el laboratorio. Sin duda, estudios en ecología química abren todo un campo para el planteamiento de métodos alternativos para el manejo de poblaciones de $O$. suberosus como una estrategia de conservación de la tortuga en el Santuario Playa La Escobilla (Oaxaca).

\section{CONCLUSIONES Y PERSPECTIVAS DE FUTURO}

El desarrollo en ecología química ha sido estimulado por el interés en conocer los detalles de las interacciones químicas entre organismos, encontrar semioquímicos con propiedades de interés humano y cómo aprovechar ese conocimiento en la conservación de la biodiversidad, así como las consecuencias evolutivas y ecológicas de éstas. Un aspecto que se requiere destacar es el esfuerzo de equipos de investigación como una parte importante en el avance de este tan complejo y particular campo de la ecología química. Así, los estudios en ecología química pueden contribuir a la conservación de la biodiversidad, demostrando lo valiosa que esta puede ser en muchos sentidos.

\section{AGRADECIMIIENTOS}

Agradezco a J.R. Verdú por la lectura crítica y comentarios al manuscrito.

\section{REFERENCIAS}

ANAYA, A.L., ESPINOSA, F.J. \& CRUZ-ORTEGA, R. 2001. Relaciones Químicas entre Organismos: Aspectos Básicos y perspectivas de su aplicación. Editorial Plaza y Valdés, S.A. de C.V. 733 pp.

BLUM, M.S. 1981. Chemical defenses of arthropods. New York: Academic Press. 562 pp.

BLUM, M.S. 1996. Semiochemical parsimony in the Arthropoda. Annu. Rev. Entomol. 41:353-374.

CAPORALE, L.H.1995. Chemicalecology: a viewfrom the pharmaceuticalindustry. Proc. Natl Acad. Sci. U.S.A. $92,75-82$.

DETTNER, K. 1987. Chemosystematics and evolution of beetle chemical defenses. Annu Rev. Entomol.32:17-48.

DICKE, M. \& SABELIS, M.W. 1988. Infochemical terminology: based on cost-benefit analysis rather than origin of compounds? Func. Ecol. 2:131-139.

ERLICH, P.R. \& RAVEN, P.H. 1965. Buterflies and plants; a study in coevolution. Evolution. 18:586-608.

HALFFTER, G., ESCOBAR, F., BAENA, ML. \& ROS, M. 2009. Distribución espacial y evaluación del daño de Omorgus suberosus (Coleoptera: Trogidae) en nidos de la tortuga marina Lepidochelys olivacea en el Santuario Playa Escobilla, Oaxaca. Informe final 63 pp

HEUSKIN, S., VERHEGGEN, F., HAUBRUGE, E., WATHELET, J.P. \& LOGNAY, G. 2011. The use of semiochemical slow-release devices in integrated pest management strategies. Biotechnol. Agron. Soc. Environ. 15(3):459-470.

HICK, A.J., LUSZNIAK, M.C. \& PICKETT, J.A. 1999. Volatile isoprenoids that control insect behaviour and development. Natural Product. Reports. 16:39-54 
HOLLIDAY, A., WALKER, F., BRODIE, E. \& FORMICA, V. 2009. Differences in defensive volatiles of the forked fungus beetle, Bolitotheruscornutus, living on two species of fungus. J. Chem. Ecol. 35:1302-1308.

LAW, J.H. \& REGNIER, F.E. 1971. Pheromones. Annual. Rev. Biochem. 40:533-548.

LÓPEZ, R.E. \& ARAGÓN, L. 1994. Programa de investigación y conservación de las Tortugas Marinas. Informe final de las actividades realizadas durante la temporada de anidación 1992-93 de la tortuga golfina (Lepidochelys olivacea) en la el Santuario Playa La Escobilla, Tonameca, Pochutla, Oaxaca. Universidad Autónoma Benito Juárez, Oaxaca
LÓPEZ-REYES, E.M. \& HARFUSH, M. 2000. Determination of the percentage of olive ridley (Lepi-dochelys olivacea) In situ nests that are affected by beetles at Escobilla beach, Mexico. p. 83 In: A. Mosier, A. Foley and B. Brost (eds). Proceedings of the Twentieth Annual Symposium on Sea Turtle Biology and Conservation. NOAA. Tech. Memo NMFS-SEFSC-477, 369 pp

NORLUND, D.A. \& LEWIS, W.J. 1976. Terminology of chemical releasing stimuli in intraspecific and interspecific interactions. J. Chem. Ecol. 2:211-220.

RUTHER, J., MEINERS, T. \&STEIDLE, J. 2002. Rich in phenomena-lacking in terms. A classification of kairomones. Chemoecology. 12:161-167 